\title{
On the Primordial Magnetic Field from Domain Walls
}

\author{
P. Cea ${ }^{1,2, *}$, and L. Tedesco ${ }^{1,2, \dagger}$ \\ ${ }^{1}$ Dipartimento di Fisica, Università di Bari, I-70126 Bari, Italy \\ ${ }^{2}$ INFN - Sezione di Bari, I-70126 Bari, Italy
}

November, 2002

\begin{abstract}
In this paper we discuss once more the zero mode contribution to the vacuum energy density. We show that a careful treatment of the zero modes leads to the conclusion that domain walls may be ferromagnetic, and could generate a magnetic field of cosmological interest.
\end{abstract}

\footnotetext{
*Electronic address: Cea@ba.infn.it

$\dagger^{\dagger}$ Electronic address: luigi.tedesco@ba.infn.it
} 
In Ref. [1] we suggested that the spontaneous generation of uniform magnetic condensate in $Q E D_{3}$ [2], [3], [4] could give rise to ferromagnetic domain walls at the electroweak phase transition. Moreover, we suggested that these domain walls generate a magnetic field at the electroweak scale which can be relevant for the generation of the primordial magnetic field [5]. In this paper we focus on some relevant points of our technique with respect to other approaches. In particular in Ref. [6], for example, the author points out that massive $(2+1)$ dimensional fermions bound to a domain wall behave diamagnetically rather than ferromagnetically. This delicate question concernes the magnetic field contribution to the fermionic vacuum free energy discussed for the first time in Ref. [2]. The aim of the present paper is twofold. First, we show that the results of [6] derive from an incorrect treatment of the fermionic zero mode contribution to the zero temperature vacuum energy density. Secondly, we show that the finite temperature calculation of Ref. [6] agrees with our previous findings [4], [7]. Moreover, if one takes into account the correct definition of an Abelian magnetic field in presence of a varying scalar field condensate, then it turns out that the classical magnetic energy is proportional to the area of the wall. This last result supports our previous proposal in Ref. [1].

The crucial point of the question is the zero temperature vacuum energy density due to massive planar fermions in presence of a constant magnetic field:

$$
E_{\mathrm{vac}}=-e B \frac{2}{\pi} \sum_{n=0}^{\infty} \sqrt{2 e B n+m^{2}} .
$$

The infinite sum in Eq. (1) needs to be regularized. Among the possible gauge-invariant choices, we employed the Schwinger proper-time regularization scheme. However, any gauge invariant regularization gives physically sensible results. Following Ref. [6] we regularize the sum by means of the gauge invariant cut-off $\exp \left(-\epsilon E_{n}^{2}\right)$, where $\epsilon$ is the regulator parameter and $E_{n}$ is the energy of the levels. We get:

$$
E_{\mathrm{vac}}^{(r)}(B)=-\frac{e B}{2 \pi} \sum_{n=0}^{\infty} \sqrt{2 e B n+m^{2}} \exp \left(-\epsilon 2 e B n-\epsilon m^{2}\right) .
$$

To evaluate the sum in Eq. (2), following Ref. [6] we use the Poisson's summation formula:

$$
\sum_{n=0}^{\infty} f(n)=\int_{\delta}^{\infty} f(x) \sum_{n=-\infty}^{\infty} \delta(x-n) d x=\sum_{k=-\infty}^{\infty} \int_{\delta}^{\infty} f(x) \exp (2 \pi i k x) d x
$$

where $\delta$ is such that $-1<\delta<0$. Now, observing that the summand in Eq. (2) is non singular at $n=0$, the author of Ref. [6]

assumes that one can set $\delta=0$. However it is easy to see that this last assumption is not valid. Indeed, if we consider the $n=0$ term in Eq. (3) :

$$
I_{\delta} \equiv \int_{\delta}^{\infty} f(x) \delta(x) d x
$$

we see that $\lim _{\delta \rightarrow 0^{-}} I_{\delta}=f(0)$, while $\lim _{\delta \rightarrow 0^{+}} I_{\delta}=0$. It is now evident that the limit $\delta \rightarrow 0$ is not harmless. Indeed, the Ref. [6] procedure is equivalent to ignore the zero mode contribution.

The correct procedure can be obtained as follows. First one must isolate the zero mode 
contribution and, then, one can apply the Poisson's summation formula to the $n \geq 1$ modes. In this way, in the weak magnetic field region we get for the vacuum energy density:

$$
\begin{aligned}
E_{\mathrm{vac}}(B)-E_{\mathrm{vac}}(0)= & -\frac{e B}{4 \pi}|m|+\frac{e^{2} B^{2}}{2 \pi^{3}|m|} \sum_{p=0}^{\infty} \frac{(-1)^{p} \zeta(2 p+2) \Gamma\left(\frac{3}{2}\right)}{\Gamma\left(\frac{1}{2}-2 p\right)}\left(\frac{e B}{\pi m^{2}}\right)^{2 p} \\
& =-\frac{e B}{4 \pi}|m|+\frac{e^{2} B^{2}}{24 \pi|m|}+\ldots .
\end{aligned}
$$

This last equation agrees with the results obtained in Ref. [2], and differs from Eq. (14) of Ref. [6] in the negative term, linear in the magnetic field. Obviously, it is the linear term which gives rise to the spontaneous magnetic condensation in $Q E D_{3}$.

Equation (5) shows that the results reported in Ref. [6], corrected to include the zero mode contributions, fully support the spontaneous generation of a uniform magnetic condensate in $Q E D_{3}$ with massive fermions. Moreover, it is worthwhile to stress that even the free energy higher temperature study of Ref. [6] is in agreement with the one reported in Ref. [4], [7]. Indeed, if one takes into account the missing linear term in the zero temperature energy density, it easy to see that Eq.(18) of Ref. [6] is in accordance with our previous conclusion that the thermal corrections, even at infinite temperature, do not modify the spontaneous generation of the magnetic condensate.

Let us now comment on the claim in Ref. [6], that the domain walls cannot be a source of the primordial magnetic field. This statement is based on the argument that the classical energy of the induced magnetic field is proportional to the volume of the box, while the contribution due to the fermion modes localized on the wall scales with the area of the wall. In this way one could obtain for the total energy of the system

$$
E(B)=L^{3} \frac{B^{2}}{2}+L^{2} f(B)
$$

where $L$ is the linear size of the system. It turns out that the above equation forgets completely the non Abelian nature of the induced magnetic field. Indeed, in the case of varying scalar field condensate the correct definition of Abelian electromagnetic field is given by the t'Hooft's Abelian projection [8]. Taking into account that in our model the Abelian part of the Abelian projected magnetic field is induced by the fermionic modes localized on the wall, it is easy to see that the Abelian projected magnetic field vanishes in the regions where the scalar condensate is constant. As a matter of fact, one can think of our field configuration in terms of a sheet of Abelian magnetic monopoles of constant surface density localized on the surface of scalar condensate zeroes. Such monopole sheet gives rise to an almost constant magnetic field perpendicular to sheet which extends over a distance of order of the wall thickness $\Delta$. Indeed it is easy to see that the appropriate expression for the Abelian magnetic field can be different from zero only in the regions where the scalar condensate varies. It is evident, now, that the Abelian projected magnetic field increases the energy of the system by the "classical energy" $\sim L^{2} \Delta \frac{B^{2}}{2}$ instead of $L^{3} \frac{B^{2}}{2}$ in Eq. (6). Thus Eq. (6) is replaced by:

$$
E(B)=L^{2} \Delta \frac{B^{2}}{2}+L^{2} f(B)
$$

which led to the conclusions of Ref. [1]. Recently, in Ref. [9] it is pointed out that due to $C$ parity arguments it cannot be spontaneous generation of magnetic field in $(3+1)$ dimensions. Our previous discussion shows that the criticism of Ref. [9] does not apply. 
Indeed, our peculiar magnetic field can be generated due to the non Abelian nature of the electroweak gauge groups where charge conjugation is no longer a symmetry.

In conclusion, it is worthwhile to stress that in the realistic case where the domain wall interacts with the plasma, the magnetic field penetrates into the plasma over a distance of the order of the penetration length $\lambda$ which is about an order of magnitude greater than $\Delta$. It follows that the estimate in Ref. [1] of the induced magnetic field at the electroweak scale $B^{*} \simeq 510^{24}$ Gauss is reduced by a factor $\sqrt{\frac{\Delta}{\lambda}} \sim 0.3$ which, however, is still of cosmological interest. However, it is worthwhile to stress that a complete treatment of the problem requires the discussion of the generation and evolution of the domain walls and their interaction with the surrounding plasma.

\section{References}

[1] P. Cea and L. Tedesco, Phys. Lett. B450 (1999) 61.

[2] P. Cea, Phys. Rev. D 32 (1985) 2785; Phys. Rev D 34 (1986) 3229.

[3] Y. Hosotani, Phys. Lett. B319 (1993) 332; Phys Rev. D 51 (1995) 2022.

[4] P. Cea and L. Tedesco, Jour. Phys.G26 (2000) 411;

[5] A. D. Dolgov, Generation of Magnetic Fields in Cosmology, hep-ph/0110293;

[6] M. B. Voloshin, Phys. Lett. B419 (2000) 311;

[7] P. Cea and L. Tedesco Phys. Lett. B425 (1998) 345.

[8] G. 't Hooft, Nucl. Phys. B79 (1974) 276; see also : T. Vachaspati, Phys. Lett. B265 (1991) 258.

[9] M. B. Voloshin, Phys. Rev. D 63 (2001) 125012. 